\title{
Prevalência do Uso de Diagnóstico Genético Pré-Implantação na Unidade Clínica de Paramiloidose do Centro Hospitalar do Porto
}

\author{
Prevalence of Use of Preimplantation Genetic Diagnosis in Unidade Clínica \\ de Paramiloidose from Centro Hospitalar do Porto
}

\author{
Kátia VALDREZ ${ }^{1,2}$, Elisabete ALVES ${ }^{1}$, Teresa COELHO ${ }^{2}$, Susana SILVA ${ }^{1}$ \\ Acta Med Port 2014 Nov-Dec;27(6):710-716
}

RESUMO

Introdução: A Polineuropatia Amiloidótica Familiar, cujo maior foco mundial é em Portugal, é reconhecida pelo Conselho Nacional de Procriação Medicamente Assistida como uma doença grave elegível para Diagnóstico Genético Pré-Implantação. Pretendemos determinar a prevalência do uso de Diagnóstico Genético Pré-Implantação nos portadores de Polineuropatia Amiloidótica Familiar seguidos na Unidade Clínica de Paramiloidose, Centro Hospitalar do Porto, e identificar os fatores associados.

Material e Métodos: Entre janeiro e maio de 2013, recrutamos sistematicamente uma amostra representativa de portadores entre os 18 e 55 anos. A análise baseia-se em 111 portadores com diagnóstico familiar prévio da doença, que referiram estar envolvidos numa tentativa de gravidez alguma vez depois de 2001. Através de questionário autoadministrado, recolhemos dados sociodemográficos e informações sobre o uso de Diagnóstico Genético Pré-Implantação. Para comparação de proporções, utilizamos o teste de qui-quadrado. Odds ratios brutos e ajustados e os respetivos intervalos de confiança de 95\% (IC 95\%) foram estimados através de regressão logística multivariada.

Resultados: A prevalência de uso de Diagnóstico Genético Pré-Implantação foi de 20,7\% (IC 95\%: 13,6-29,5). Após ajuste, o rendimento familiar superior a $1000 € /$ mês $(O R=11,87$; IC 95\% 2,87-49,15) associou-se diretamente ao uso Diagnóstico Genético PréImplantação, enquanto portadores com diagnóstico individual $(O R=0,15$; IC 95\% 0,04-0,57) e filhos nascidos após 2001 (OR = 0,07; IC $95 \% 0,02-0,32$ ) revelaram uma prevalência de uso significativamente menor do que aqueles com diagnóstico individual e filhos nascidos antes de 2001.

Discussão: A baixa prevalência de uso de Diagnóstico Genético Pré-Implantação, bem como a utilização menos frequente da técnica por aqueles com um rendimento familiar mais baixo evidenciam a importância de melhorar a acessibilidade ao Diagnóstico Genético Pré-Implantação no caso da Polineuropatia Amiloidótica Familiar.

Conclusão: Este trabalho contribui para o aumento da sensibilidade dos profissionais de saúde em torno do uso e da acessibilidade ao Diagnóstico Genético Pré-Implantação entre os portadores de Polineuropatia Amiloidótica Familiar.

Palavras-chave: Diagnóstico Genético Pré-Implantação; Polineuropatia Amiloidótica Familiar; Testes Genéticos; Procriação Medicamente Assistida.

\section{ABSTRACT}

Introduction: The Familial Amyloid Polyneuropathy, with the world's largest focus in Portugal, is recognized by the National Board of Assisted Reproductive Technologies as a serious disease eligible for Preimplantation Genetic Diagnosis. This study aims to determine the prevalence of the use of Preimplantation Genetic Diagnosis in FAP carriers followed in Unidade Clínica de Paramiloidose, Centro Hospitalar do Porto, and to identify the associated factors.

Material and Methods: Between January and May 2013, a representative sample of Portuguese Familial Amyloid Polyneuropathy carriers, aged between 18 and 55 years, was systematically recruited. The analysis is based on 111 carriers with previous familial diagnosis, who reported having ever tried to get pregnant after 2001. Data on sociodemographic characteristics and use of Preimplantation Genetic Diagnosis were collected through a self-administered questionnaire. Proportions were compared using the chi-square test. Crude and adjusted odds ratios (OR) and the respective confidence intervals of $95 \%(95 \% \mathrm{Cl})$ were estimated using multivariate logistic regression.

Results: The prevalence of use of Preimplantation Genetic Diagnosis was $20.7 \%$ (95\% Cl: 13.6-29.5). After adjustment, a household income above $1000 € /$ month ( $\mathrm{OR}=11.87 ; 95 \% \mathrm{Cl} 2.87-49.15)$ was directly associated with the use of Preimplantation Genetic Diagnosis, while carriers with an individual diagnosis $(\mathrm{OR}=0.15 ; 95 \% \mathrm{Cl} 0.04-0.57)$ and children born after $2001(\mathrm{OR}=0.07 ; 95 \% \mathrm{Cl} 0.02-$ 0.32) revealed a prevalence of use significantly lower than those with a individual diagnosis and children born before 2001.

Discussion: The low prevalence of use of Preimplantation Genetic Diagnosis, as well as the less frequent use of the technique by those with a lower household income, shows the importance of improving access to Preimplantation Genetic Diagnosis in the case of Familial Amyloid Polyneuropathy.

Conclusion: This work contributes to increase the sensitivity of health professionals around the use and accessibility to Preimplantation Genetic Diagnosis among Familial Amyloid Polyneuropathy carriers.

Keywords: Preimplantation Diagnosis; Amyloid Neuropathies, Familial; Genetic Testing; Assisted Reproductive Technologies.

\section{INTRODUÇÃo}

Portugal regista o maior foco mundial de Polineuropatia Amiloidótica Familiar (PAF), também conhecida como

paramiloidose ou 'doença dos pezinhos'. ${ }^{1}$ Esta doença hereditária de transmissão autossómica dominante (ou seja,

1. Instituto de Saúde Pública da Universidade do Porto (ISPUP). Departamento de Epidemiologia Clínica, Medicina Preditiva e Saúde Pública. Faculdade de Medicina. Universidade do Porto. Porto. Portugal.

2. Serviço de Neurologia. Unidade Clínica de Paramiloidose. Centro Hospitalar do Porto. Porto. Portugal.

Recebido: 07 de Novembro de 2013 - Aceite: 24 de Fevereiro de 2014 | Copyright @ Ordem dos Médicos 2014 
a probabilidade de transmitir o gene à descendência é de $50 \%$ ) surge habitualmente entre os 25 e os 35 anos e tem um atingimento multi-sistémico, tratando-se de uma doença crónica gradualmente incapacitante. Em 1976, a PAF foi considerada um problema de saúde pública no nosso país, atendendo ao número de portadores (431 casos registados até 1976) e à gravidade das suas manifestações incapacitantes. $^{2}$ De acordo com o mapeamento mais recente dos portadores de PAF em Portugal, levado a cabo em março de 2011 pela Unidade Clínica de Paramiloidose (UCP) do Hospital de Santo António (HSA), integrado no Centro Hospitalar do Porto (CHP), estima-se a existência de 1657 casos de PAF neste país. Destes, 54\% são mulheres, $80 \%$ têm menos de 55 anos e $27 \%$ são assintomáticos. Os municípios com maior prevalência da doença são Póvoa de Varzim/Vila do Conde, Braga e Barcelos.

A transmissão desta doença pode ser evitada se os portadores decidirem não ter filhos biológicos; se optarem pela Interrupção Voluntária da Gravidez (IVG) no caso do Diagnóstico Pré-Natal (DPN) confirmar que o feto é afetado pela doença; ou se recorrerem ao Diagnóstico Genético Pré-Implantação (DGPI), método de diagnóstico embrionário que permite detetar a PAF em embriões antes de estes serem transferidos para o útero. Atendendo à importância atribuída à parentalidade genética na construção da identidade humana, individual e coletiva, em particular na sociedade portuguesa, ${ }^{3}$ e aos custos emocionais e de saúde implicados na IVG ${ }^{4}$ aos portadores de PAF é proporcionada a possibilidade de utilizar o DGPI quando e se pretenderem concretizar o desejo de serem pais. ${ }^{5}$

O DGPI está disponível em Portugal desde $1997^{6}$ e o primeiro protocolo que contempla a sua aplicação à PAF foi publicado em 2001. ${ }^{7}$ Desde dezembro de 2012 que a PAF é reconhecida pelo Conselho Nacional de Procriação Medicamente Assistida (CNPMA) como uma das doenças graves elegíveis para $\mathrm{DGPI}^{8}$, reunindo todos os requisitos estabelecidos para tal classificação: a PAF causa sofrimento significativo e/ou morte prematura; a causalidade genética está estabelecida; existe uma relação clara entre a presença da alteração genética a pesquisar e a existência (ou risco elevado) de doença nos indivíduos portadores; há a possibilidade técnica de identificação da alteração genética nos embriões ou ovócitos; a fiabilidade do diagnóstico genético é superior a $90 \%$; e o risco de transmissão da doença genética à descendência é significativamente superior ao verificado na população geral. ${ }^{9}$

Este reconhecimento pode contribuir para melhorar a acessibilidade às técnicas de PMA, um dos objetivos estabelecidos pela Organização Mundial de Saúde (OMS) para este milénio. ${ }^{10-12}$ Tal propósito tem sido visível noutras propostas do Governo Português, desde a publicação da primeira lei que regula a utilização destas técnicas em 2006 até à estandardização dos respetivos custos e avaliação da qualidade nos anos subsequentes. ${ }^{13,14}$ Por exemplo, a realização do DGPI tem de ser precedida por uma consulta de aconselhamento genético, conduzida por um profissional da especialidade de genética médica, e a sua execução é obrigatoriamente consentida pelos dois elementos do casal. ${ }^{9}$ Adicionalmente, a aplicação do DGPI deve ser orientada por um médico especialista, em articulação com uma equipa multidisciplinar que inclua especialistas em medicina da reprodução, embriologistas, médicos geneticistas, citogeneticistas e geneticistas moleculares. ${ }^{15}$

O CNPMA publicou, em fevereiro de 2013, orientações sobre o DGPI. Estas contemplam a inclusão de informações sobre o número de tratamentos realizados e respetivas indicações, assim como os resultados do DPN confirmatório, quando realizado, nos relatórios anuais a elaborar pelos Centros de PMA. ${ }^{9}$ No entanto, neste momento não existe um registo nacional de dados sobre o uso de DGPI por portadores de PAF. Assim, o presente estudo tem como objetivo determinar a prevalência do uso de DGPI nos portadores de Paramiloidose seguidos na UCP do CHP e identificar os fatores que lhe estão associados.

\section{MATERIAL E MÉTODOS}

Realizamos este estudo na UCP do CHP. O protocolo de estudo foi aprovado pelo Conselho de Administração do Centro Hospitalar do Porto, após parecer favorável da Comissão de Ética para a Saúde e do Gabinete Coordenador de Investigação, bem como da Direção Clínica. Todos os participantes assinaram uma declaração de consentimento informado na altura de preenchimento do questionário.

Em março de 2011, esta Unidade procedeu a um levantamento do número de portadores de PAF em Portugal, com base em informações recolhidas junto das seguintes instituições e profissionais de saúde: unidades clínicas com seguimento de portadores de PAF no Porto e Lisboa; unidades de transplantação hepática em Lisboa, Coimbra e Porto; médicos responsáveis pelas consultas organizadas de PAF nos concelhos de Covilhã, Seia, Figueira da Foz, Barcelos e Esposende; Associação de Paramiloidose e os seus núcleos locais de doentes. De acordo com estes dados, estima-se a existência de 1657 casos de PAF no nosso país, $64 \%$ ( $n=1055)$ dos quais são seguidos na UCP do CHP (relatório não publicado).

Todos os portadores seguidos na UCP do CHP com idades compreendidas entre os 18 e os 55 anos foram considerados elegíveis para participar no presente estudo. Em dezembro de 2012, encontravam-se nesta condição 913 indivíduos. Entre janeiro e maio de 2013, 271 portadores de PAF foram consecutivamente convidados a integrar o estudo, garantindo um nível de significância de 5\% e um poder estatístico de $95 \%$. Recrutamos sistematicamente uma amostra representativa de 266 portadores, correspondente a uma proporção de participação de 98,2\%.

Os portadores foram recrutados nos dias em que se deslocavam à UCP para efetuar consultas de rotina previamente agendadas em várias especialidades (neurologia, nefrologia, psiquiatria e oftalmologia, entre outras). Durante o período do estudo foram destacados dois profissionais de saúde desta Unidade para convidar os portadores elegíveis a participar no estudo, de acordo com o funcionamento normal e a disponibilidade do serviço, assegurando desta 
forma o recrutamento diário, consecutivo e sistemático dos participantes. Estes profissionais entregaram um folheto explicativo do projeto a cada portador elegível, solicitando a respetiva colaboração e esclarecendo qualquer dúvida.

Com base num questionário autoadministrado construído pela equipa de investigação, recolhemos dados demográficos e socioeconómicos dos participantes, informações sobre a sua história reprodutiva e obstétrica e sobre o uso e perceções acerca do DGPI (incluindo as fontes de informação e os motivos para usar e não usar esta técnica). Incluímos neste estudo o sexo, a idade (categorizada em $<35$ e $\geq 35$ anos), o local de residência, o estatuto marital (categorizado em solteiro(a) vs. casado(a), união de facto, divorciado(a) ou viúvo(a)), o grau de escolaridade (categorizado em $\leq 12^{\circ}$ ano e $>12^{\circ}$ ano), a situação profissional (categorizada em empregado vs. desempregado, doméstico, trabalhador não remunerado, estudante ou reformado) o rendimento mensal líquido do agregado familiar (categorizado em $\leq 1000 € \mathrm{e}>1000 €$ ), a classe social subjetiva (baixa/média baixa vs. alta/média alta), a religião (categorizada em católica, protestante ou outra cristã vs. nenhuma religião), o número de filhos biológicos nascidos após 31 de dezembro de 2001 e o uso de DPN. O uso de DGPI foi considerado positivo quando os participantes referiram a sua utilização em pelo menos uma das tentativas para alcançar uma gravidez. $\mathrm{O}$ ano do diagnóstico individual e familiar de PAF foi obtido na base de dados da UCP, do CHP.

A presente análise baseia-se em 111 participantes, cuja trajetória de vida incluía a possibilidade de terem sido confrontados com a efetiva decisão de usar ou não usar DGPI. Trata-se de indivíduos com diagnóstico familiar prévio da doença, que referiram ter estado alguma vez envolvidos numa tentativa de gravidez depois de 31 de dezembro de 2001, ano em que foi publicado o primeiro protocolo que contempla a aplicação do DGPI à PAF. ${ }^{7}$ Destes, 107 possuíam informação disponível para todas as variáveis incluídas no modelo final.

A análise estatística foi efetuada com recurso ao programa STATA 11.0 (College Station, TX, 2009). A prevalência de uso de DGPI é apresentada com o respetivo intervalo de confiança a 95\% (IC 95\%). O teste de qui-quadrado foi usado para comparação de proporções. Estimámos odds ratios (OR) brutos e ajustados com os respetivos IC 95\% por regressão logística, para avaliar os fatores associados ao uso do DGPI. Os modelos multivariados foram construídos partindo das variáveis cuja associação com o uso do DGPI era estatisticamente significativa nesta amostra ou que os investigadores consideraram ser potenciais confundidores da associação. As variáveis que na análise multivariada não mantinham associação independente com a utilização do DGPI e não confundiam o efeito das restantes foram excluídas do modelo final.

\section{RESULTADOS}

No presente estudo, $61,3 \%$ dos participantes pertenciam ao sexo feminino e metade tinham 35 anos de idade ou mais (Tabela 1). Cerca de $70 \%$ residiam nos distritos de Braga ou Porto e mais de $90 \%$ tinham já estado envolvidos numa relação de coabitação. Enquanto $46 \%$ possuíam mais de 12 anos de escolaridade, $76,4 \%$ estavam empregados e aproximadamente $42 \%$ referiram um rendimento familiar superior a $1000 €$ por mês, apenas $13,8 \%$ dos participantes se percecionaram como pertencendo às classes sociais alta ou média alta. Mais de $95 \%$ dos portadores referiram uma crença religiosa, sendo a religião católica a mais frequentemente reportada $(88,3 \%)$. Cerca de $60 \%$ e $85 \%$ tiveram um diagnóstico de doença e referiram o nascimento de filhos biológicos após 2001, respetivamente. O recurso ao DPN foi reportado por um quinto dos participantes.

Dos 111 portadores elegíveis para DGPI, ou seja, que referiram ter estado alguma vez envolvidos numa tentativa de gravidez depois de 31 de dezembro de 2001 e com diagnóstico familiar da doença, a prevalência de uso de DGPI foi de $20,7 \%$ (IC 95\% 13,6-29,5). Os portadores que recorreram ao DGPI possuíam frequentemente níveis de escolaridade superiores ao $12^{\circ}$ ano $(73,9 \%$ vs. $38,6 \% ; p=0,003)$, rendimentos familiares acima de $1000 € /$ mês $(72,7 \%$ vs. $33,7 \% ; p=0,001$ ) e percecionavam-se mais frequentemente como pertencendo às classes sociais alta ou média alta $(27,3 \%$ vs.10,3\%; $p=0,039)$ do que aqueles que nunca recorreram ao DGPI. Portadores com diagnóstico individual posterior a $2001(39,1 \%$ vs. $65,9 \%, p=0,019)$ e com filhos nascidos após 2001 (65,2\% vs. 90,9\%, $p=0,002)$ revelaram-se estatisticamente menos propensos a utilizar o DGPI. Não se verificaram diferenças estatisticamente significativas entre utilizadores e não utilizadores relativamente ao sexo, idade, local de residência, estatuto marital, situação profissional, religião e utilização de DPN (Tabela 1).

$\mathrm{Na}$ Tabela 2 apresentamos os principais fatores associados à utilização do DPGI por portadores de PAF. Após ajuste para a idade, sexo e todas as variáveis significativamente associadas com a utilização do DGPI, apenas as associações descritas para o rendimento do agregado familiar, o ano do diagnóstico individual e o nascimento de filhos após 2001 se mantiveram estatisticamente significativas. Assim, os efeitos estatísticos descritos para a escolaridade e a classe social subjetiva parecem ser explicados pelo efeito do rendimento, que se destaca como o indicador socioeconómico mais relevante para o uso de DGPI. Portadores com diagnóstico individual de PAF após 2001 $(O R=0,15$; IC 95\% 0,04-0,57) e com filhos nascidos após esse ano $(O R=0,07$; IC 95\% 0,02-0,32) revelaram uma prevalência de uso significativamente menor do que aqueles com diagnóstico individual e filhos nascidos antes de 2001, respetivamente. O rendimento familiar associou-se diretamente ao uso do DGPI por portadores de PAF (OR = 11,87 ; IC 95\% 2,87-49,15).

\section{DISCUSSÃO}

Este estudo é, no melhor do nosso conhecimento, o primeiro a avaliar a prevalência de uso de DGPI por porta- 


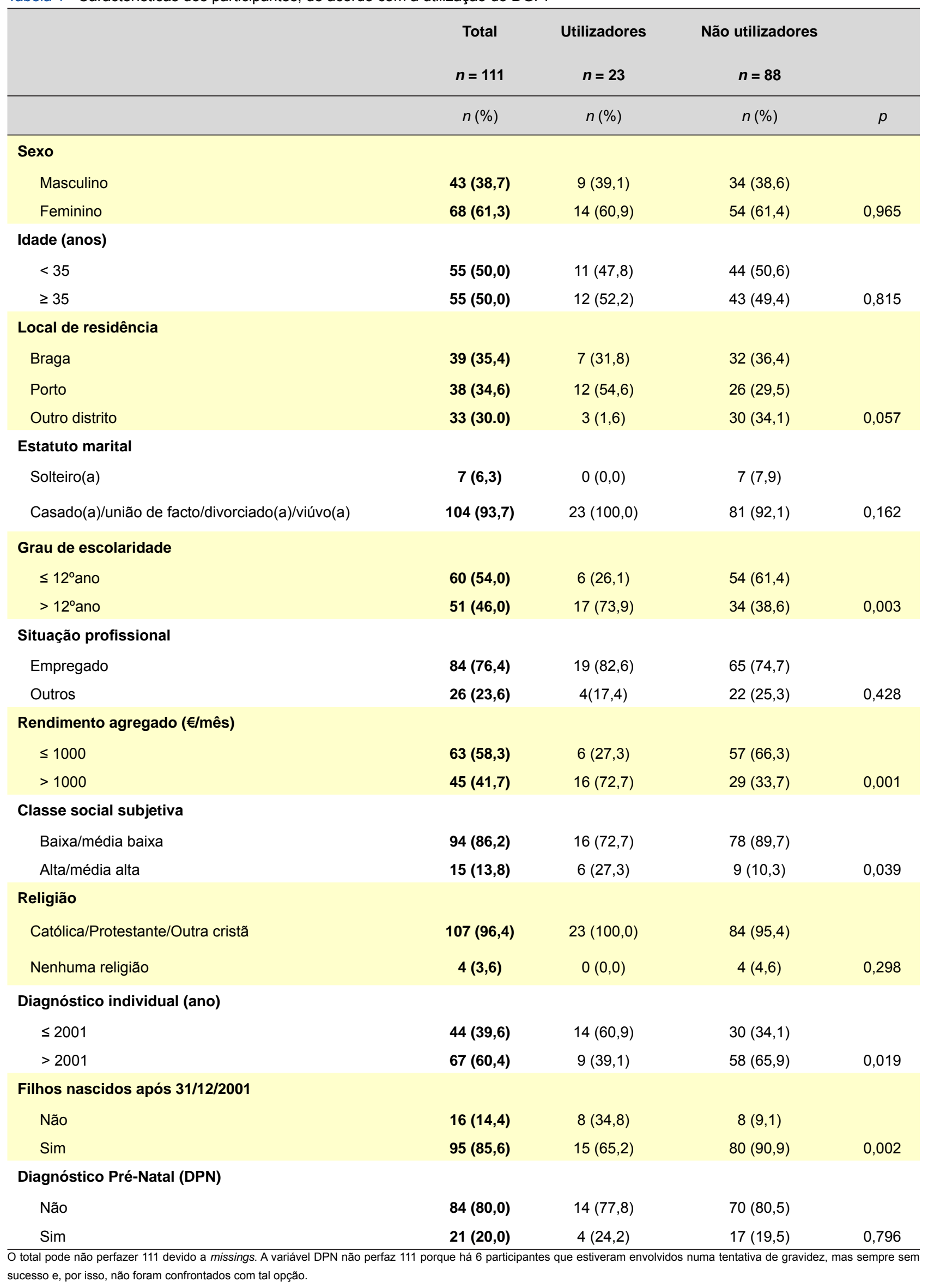


Tabela 2 - Fatores associados à utilização do DGPI por portadores de PAF $(n=107)$
OR bruto
(IC 95\%)
OR ajustado*
(IC 95\%)

Diagnóstico individual (ano)

$$
\leq 2001
$$

1

$>2001$

$0,36(0,14-0,94)$

$0,15(0,04-0,57)$

Filhos nascidos após 31/12/2001

Não

1

$\operatorname{Sim}$

$0,18(0,06-0,56)$

1

$0,07(0,02-0,32)$

Rendimento agregado (€/mês)

$\leq 1000$

1

$>1000$

$5,15(1,82-14,57)$

$11,87(2,87-49,15)$

C - intervalo de confiança; $O R$ - Odds ratio * Ajustado para a idade, sexo e todas as variáveis da tabela.

dores de PAF em Portugal. Tendo em conta que Portugal é o país mais atingido por esta doença, cujas manifestações clínicas são graves, incapacitantes e condicionam a qualidade de vida e a sobrevida dos indivíduos afetados, conhecer a prevalência de utilização do DGPI é fundamental. Os nossos resultados mostraram que um quinto dos portadores de PAF referiu ter recorrido ao DGPI, e que o rendimento familiar mais elevado se associou diretamente ao uso desta técnica, enquanto o diagnóstico individual e o nascimento de filhos após 2001 se revelaram inversamente associados ao recurso ao DGPI. Os únicos dados divulgados publicamente sobre esta questão foram apresentados, oralmente, no European Advanced Postgraduate Course on Transthyretin Amyloidosis, realizado em maio de 2013 no CHP (comunicação oral de Filipa Carvalho, dados não publicados). Concluiu-se que, entre janeiro de $1999 \mathrm{e}$ maio de 2013, 62 homens e 36 mulheres portadores de PAF realizaram DGPI no Centro Hospitalar de S. João, o único a proporcionar tal serviço no setor público. Num total de 186 ciclos de DGPI (136 ciclos com transferência de embriões e 50 ciclos sem transferência), confirmaram-se 40 gravidezes clínicas (28 gravidezes únicas, sete gravidezes múltiplas, três em curso e dois abortos), das quais nasceram 43 crianças. Trata-se, assim, de 98 portadores que usaram DGPI nesta unidade, num contexto em que se estima a existência, em março de 2011, de 1657 portadores de PAF em Portugal, 64\% $(n=1055)$ dos quais são seguidos na UCP, do CHP. Os nossos resultados sobre a prevalência de uso de DGPI na UCP do CHP estão em aparente alinhamento com estes dados, se considerarmos que os nossos participantes recorreram a DGPI, quer nesta instituição pública, quer em centros de PMA privados.

Os resultados apresentados neste estudo devem ser interpretados à luz do contexto sociocultural, político e económico que enquadra o acesso a estas técnicas, e em particular ao DGPI. O Estado proporciona aos doentes o acesso gratuito, e a título devolutivo, do material clínico de apoio e a comparticipação total de medicamentos, desde que os portadores sofram de uma incapacidade funcional igual ou superior a $70 \% .{ }^{16,17}$ No entanto, o atual financiamento público de DGPI apenas inclui casais cujo elemento feminino não ultrapasse os 40 anos e não tenham mais de um filho em comum no momento de realização da técnica, ${ }^{18}$ e estes tratamentos não são cobertos por seguros de saúde privados. O preço definido pela Portaria $n{ }^{\circ}$ 67/2011 para um tratamento com recurso a injeção intracitoplasmática de espermatozoides (ICSI) é de $2613 €,{ }^{19}$ valor mais baixo do que é praticado, por exemplo, num centro privado que estabelece o montante de $4200 €$ para um ciclo completo de ICSI, acrescendo $450 €$ para o DGPI através de biópsia embrionária. ${ }^{20}$ Neste contexto, estes fatores poderão explicar a utilização mais frequente de DGPI por portadores com um rendimento familiar mais elevado.

Esta conclusão é suportada pelos resultados evidenciados numa revisão sistemática recente sobre os fatores que influenciam o processo de decisão de casais quanto à utilização ou não do DGPI, ${ }^{21}$ que salienta a importância da avaliação cognitiva em torno dos custos financeiros envolvidos na realização de DGPI. Esta avaliação é, no entanto, ponderada em articulação com fatores que não foram avaliados no âmbito deste estudo, nomeadamente o tempo de espera estimado para concretizar uma gravidez e as informações complexas e incertas sobre as taxas relativamente baixas de sucesso e a perceção dos riscos envolvidos nos procedimentos médicos, a curto e longo prazo. ${ }^{22,23}$ Também as respostas emocionais associadas à dor e ao sofrimento e os significados atribuídos à felicidade desempenham um papel importante neste processo de tomada de decisão, ${ }^{24}$ assim como as atitudes e representações sociais sobre o estatuto do embrião e a aceitabilidade moral do uso de técnicas que visam prevenir a doença. ${ }^{25,26} \mathrm{~A}$ decisão dos potenciais utilizadores é ainda influenciada por fatores como o tempo necessário para organizar, programar e participar nas consultas e exames médicos, bem como o número e tipo de sessões de educação e aconselhamento com profissionais de saúde (p.e., geneticistas e psicólogos). ${ }^{27}$ 
Assim, a identificação dos principais fatores que influenciam a decisão quanto à utilização de DGPI evidencia a complexidade, dinamismo e interatividade do processamento de informação neste domínio, assim como a heterogeneidade dos significados atribuídos pelos potenciais utilizadores ao risco genético nas suas trajetórias reprodutivas. ${ }^{28}$ Estes elementos são fundamentais para explicar o facto de um diagnóstico individual e ter filhos antes de 2001 surgirem como fatores associados à utilização de DGPI por portadores de PAF. Tais portadores poderão ter identificado a sua condição de risco ou estar envolvidos em trajetórias ativas de parentalidade há mais tempo, beneficiando, por isso, de mais oportunidades para conhecer a técnica e para refletir sobre as diferentes opções reprodutivas à medida que têm mais consciência das consequências da doença.

É de realçar que todos os portadores com diagnóstico individual posterior a 2001 tinham um diagnóstico familiar da doença quando se envolveram numa tentativa de estabelecer uma gravidez, o que poderá revelar uma tentativa mais ou menos explícita de não querer conhecer os resultados do diagnóstico individual antes de conceber uma criança ou a perceção da PAF como uma herança familiar. Por outro lado, a não utilização de DGPI pode estar associada a preocupações quanto ao estatuto do embrião e à possibilidade desta técnica promover a estigmatização, intolerância e discriminação dos portadores. ${ }^{25,26,29}$ No entanto, estas razões só muito raramente foram escolhidas pelos participantes para justificar a não utilização de DGPI, realçando-se os custos financeiros, a desconfiança da técnica e o tempo para engravidar como os principais motivos para tal opção. ${ }^{30}$ Importa, assim, promover a acessibilidade ao DGPI, bem como investir em campanhas de sensibilização e informação que disseminem conhecimento preciso e consistente sobre esta técnica, em particular entre os portadores de PAF.

\section{REFERÊNCIAS}

1. Sousa A. Epidemilogia genética da polineuropatia amiloidótica familiar. Sinapse. 2006;6:74-9.

2. Freitas A. Aspectos clínicos da polineuripatia amiloidótica familiar tipo andrade. Boletim do Hospital. 1976;1:17-25.

3. Silva $S$, Machado $H$. The construction of meaning by experts and would-be parents in assisted reproductive technology. Sociol Health IIIn. 2011;33:853-68

4. Canário A. Aspetos éticos e psicológicos do abortamento no casal. Porto: Faculdade de Medicina da Universidade do Porto: Tese de Mestrado; 2009.

5. Associação Portuguesa de Paramiloidose [homepage na Internet]. Ter um filho sem medo que sofra de mal incurável; [consultado 2013 Nov 7]. Disponível em: http://www.paramiloidose.com/sm20.15567/pag4935. html.

6. Projeto de Lei n. ${ }^{\circ 140 / X}$. Diagnóstico Genético Pré-Implantação e Intervenções na Linha Germinativa; 2005; [consultado 2013 Nov 7]. Disponível em: http://app.parlamento.pt/webutils/docs/doc.pdf?path=61 48523063446f764c3246795a5868774d546f334e7a67774c325276593 342734c576c75615668305a586776634770734d5451774c5667755a47 396a\&fich=pjl140-X.doc\&Inline=true.

7. Carvalho F, Sousa M, Fernandes S, Silva J, Saraiva MJ, Barros A. Preimplantation genetic diagnosis for familial amyloidotic polyneuropathy. Prenat Diagn. 2001;21:1093-9.

8. Conselho Nacional de Procriação Medicamente Assistida [homepage na Internet]. Lista de situações para as quais o pedido prévio de au-

\section{CONCLUSÃO}

A baixa prevalência de uso de DGPI por portadores de PAF salienta a necessidade de compreender de que forma os portadores processam a informação e avaliam o risco no contexto do DGPI, para sustentar intervenções que visem aumentar a adesão a esta técnica. Ao mesmo tempo, a utilização menos frequente de DGPI por portadores com um rendimento familiar mais baixo evidencia a importância de melhorar a acessibilidade ao DGPI no caso da PAF, com o intuito de evitar a disseminação da doença e diminuir desigualdades no acesso e utilização de cuidados de saúde.

\section{AGRADECIMENTOS}

Os autores gostariam de agradecer a todos os portadores de PAF que integraram o estudo pela essencial colaboração e a todos os profissionais de saúde da Unidade Clínica de Paramiloidose que participaram na recolha de dados pelo tempo disponibilizado e profissionalismo revelado.

\section{CONFLITO DE INTERESSES}

Os autores declaram que não houve conflito de interesses na realização deste trabalho.

\section{FONTES DE FINANCIAMENTO}

Este trabalho resulta da dissertação de Mestrado em Saúde Pública de Kátia Valdrez, defendida na Faculdade de Medicina da Universidade do Porto, e é financiado por Fundos FEDER através do Programa Operacional Fatores de Competitividade - COMPETE e por Fundos Nacionais através da FCT - Fundação para a Ciência e Tecnologia no âmbito do projeto FCOMP-01-0124-FEDER-014453 (FCT PTDC/CS-ECS/110220/2009), e pelo programa Investigador FCT (IF/00956/2013).

torização para DGPI será dispensado; 2012 [consultado 2013 Nov 7]. Disponível em: http://www.cnpma.org.pt/Docs/DGPI_Lista_DEZEMBRO2012.pdf.

9. Conselho Nacional de Procriação Medicamente Assistida [homepage na Internet]. Diagnóstico genético pré-implantação e rastreio de aneuploidias pré-implantação; 2013 [consultado 2013 Nov 7]. Disponível em: http://www.cnpma.org.pt/Docs/Orienta\%C3\%A7\%C3\%B5es\%20 DGPI\%20FEV2013.pdf.

10. Vayena E, Rowe P, Griffin D, editors. Current practices and controversies in assisted reproduction. Geneve: World Health Organization; 2002.

11. Pennings G, Wert G, Shenfield F, Cohen J, Tarlatzis B, Devroey P. ESHRE Task Force on Ethics and Law 14: equity of access to assisted reproductive technology. Hum Reprod. 2008;23:772-4.

12. Ombelet $W$. False perceptions and common misunderstandings surrounding the subject of infertility in developing countries. ESHRE Monographs. 2008;1:8-11.

13. Silva S, Barros H. Perspectives on access to in vitro fertilization in portugal. Rev Saude Publica. 2012;46:344-50.

14. Ministério da Saúde. Despacho $n^{\circ} 14788 / 2008$. Diário da República $2^{\mathrm{a}}$ série;102: 23832-3; 2008; [consultado 2013 Nov 7]. Disponível em: http://www.portaldasaude.pt/NR/rdonlyres/71BF02C8-2F2F-40DBA8F5-66F9EEE478C9/0/2383223833.pdf.

15. Assembleia da República. Lei $n^{\circ} 32 / 2006$. Diário da República, $1^{\text {a }}$ série;143:5245-50; 2006; [consultado 2013 Nov 7]. Disponível em: http:// dre.pt/pdf1s/2006/07/14300/52455250.pdf. 
16. Gabinete do Secretário de Estado da Saúde. Despacho n 4521/2001. Diário da República, $2^{\mathrm{a}}$ série; 54:4140; 2001; [consultado 2013 Nov 7]. Disponível em: http://www.dre.pt/pdf2s/2001\%5C03\%5C054000000\% 5C0414004140.pdf

17. Assembleia da República. Lei $\mathrm{n}^{\circ} 1 / 89$. Diário da República, $1^{\mathrm{a}}$ série; 26:395-6; 1989; [consultado 2013 Nov 7]. Disponível em: http://dre.pt/ pdf1s/1989/01/02600/03950396.pdf.

18. Administração Central do Sistema de Saúde. Circular Normativa $n^{\circ}$ 18/2011. 2011; [consultado 2013 Nov 7]. Disponível em: http://www. cnpma.org.pt/Docs/Legislacao_CircularACSS_18_2011.pdf.

19. Ministério da Saúde. Portaria $n^{\circ}$ 67/2011. Diário da República, $1^{\text {a }}$ série; 25: 681-2; 2011; [consultado 2013 Nov 7]. Disponível em: http:// www.sg.min-saude.pt/NR/rdonlyres/F1071041-A28D-4B06-8CB5D640D1D60D80/25595/Portaria67 2011.pdf.

20. Centro de Genética da Reprodução Prof. Alberto Barros [homepage na Internet]. Tabela geral de preços; [consultado 2013 Nov 7]. Disponível em: http://www.cgrabarros.pt/MOD\%20074\%20R05.pdf.

21. Hershberger P, Pierce P. Conceptualizing couples' decision making in PGD: emerging cognitive, emotional, and moral dimensions. Patient Educ Couns. 2010;81:53-62.

22. Lavery S, Aurell R, Turner C, Castello C, Veiga A, Barri P, et al. Preimplantation genetic diagnosis: patients' experiences and attitudes. Hum
Reprod. 2002;17:2464-7.

23. Pergament E. Preimplantation diagnosis: a patient perspective. Prenat Diagn. 1991;11:493-500.

24. Sanfey A. Decision neuroscience: new directions in studies of judgment and decision making. Curr Dir Psychol Sci. 2007;16:151-5.

25. Snowdon C, Green J. Preimplantation diagnosis and other reproductive options: attitudes of male and female carriers of recessive disorders. Hum Reprod. 1997;12:341-50.

26. Teles N. Diagnóstico genético pré-implantação: aspetos técnicos e considerações éticas. Acta Med Port. 2011;24:987-96.

27. Zeiler K. Reproductive autonomous choice - a cherished illusion? Reproductive autonomy examined in the context of preimplantation genetic diagnosis. Med Health Care Philos. 2004;7:175-83.

28. Hershberger P, Gallo A, Kavanaugh K, Olshansky E, Schwartz A, Tur-Kaspa I. The decision-making process of genetically at-risk couples considering preimplantation genetic diagnosis: initial findings from a grounded theory study. Soc Sci Med. 2012;74:1536-43.

29. Savulescu J. In defence of procreative beneficence. J Med Ethics. 2007;33:284-8.

30. Valdrez K, Silva S, Coelho T, Alves E. Awareness and motives for use and non-use of preimplantation genetic diagnosis in familial amyloid polyneuropathy mutation carriers. Prenat Diagn. 2014;34:886-92. 
Kátia VALDREZ, Elisabete ALVES, Teresa COELHO, Susana SILVA

\section{Prevalência do Uso de Diagnóstico Genético Pré-Implantação na Unidade Clínica de Paramiloidose do Centro Hospitalar do Porto}

Acta Med Port 2014:27:710-716

Publicado pela Acta Médica Portuguesa, a Revista Científica da Ordem dos Médicos

Av. Almirante Gago Coutinho, 151

1749-084 Lisboa, Portugal.

Tel: +351218428215

E-mail: submissao@actamedicaportuguesa.com

www.actamedicaportuguesa.com

ISSN:0870-399X | e-ISSN: 1646-0758

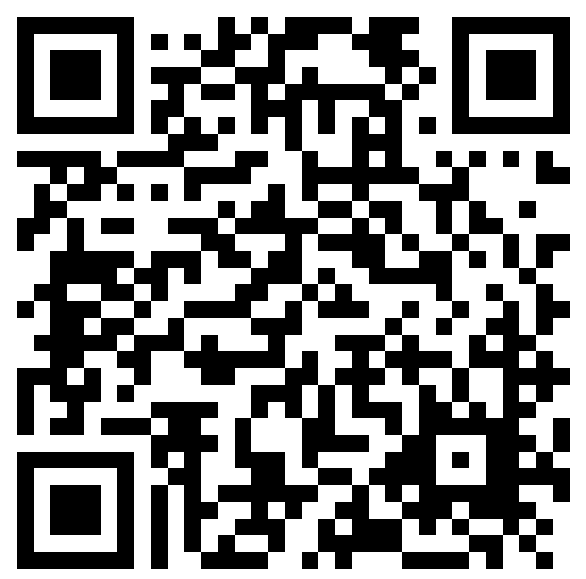

